\title{
Mortality Risk and Its Association with Geriatric Domain Deficits in Older Outpatients: The Amsterdam Ageing Cohort
}

\author{
Hanneke Frederica Maria Rhodius-Meester ${ }^{a}$ b Sara A.J. van de Schraaf ${ }^{a}$ \\ Mike J.L. Peters ${ }^{a} \quad$ Emma E.F. Kleipool ${ }^{\mathrm{a}}$ Marijke C. Trappenburga, c \\ Majon Muller ${ }^{\mathrm{a}}$
}

aDepartment of Internal Medicine, Geriatric Medicine Section, Vrije Universiteit Amsterdam, Amsterdam UMC, Amsterdam, The Netherlands; ${ }^{b}$ Alzheimer Center Amsterdam, Department of Neurology, Amsterdam Neuroscience, Vrije Universiteit Amsterdam, Amsterdam UMC, Amsterdam, The Netherlands; 'Department of Internal Medicine, Amstelland Hospital, Amstelveen, The Netherlands

\section{Keywords \\ Comprehensive geriatric assessment · Geriatric domain deficits · Mortality · Outpatient · Older people}

\begin{abstract}
Introduction: In older patients, life expectancy is determined by a complex interaction of multiple geriatric domains. A comprehensive geriatric assessment (CGA) captures different geriatric domains. Yet, if and how components of the CGA are related to mortality in an outpatient geriatric setting is unknown. In the Amsterdam Ageing Cohort, we therefore studied distribution and accumulation of geriatric domain deficits in relation to mortality. Methods: All patients received a CGA as part of standard care, independent of referral reason. We summarized deficits on the CGA, using predefined cutoffs, in 5 geriatric domains: somatic, mental, nutritional, physical, and social domain. Information on mortality was obtained from the Dutch municipal register. We used age- and sex-adjusted Cox proportional hazards analyses to relate the separate domains and accumulation of impaired domains to overall mortality. $\boldsymbol{R} \boldsymbol{e}$ sults: From the 1,055 geriatric outpatients (53\% female; age $79 \pm 7$ years), 172 patients (16\%) had died after $1.7 \pm 1.1$
\end{abstract}

karger@karger.com www.karger.com/ger

Karger $\stackrel{\text { ' }}{=}$

GOPEN ACCESS
(C) 2021 The Author(s)

Published by S. Karger AG, Basel

This is an Open Access article licensed under the Creative Common Attribution-NonCommercial-4.0 International License (CC BY-NC) (http://www.karger.com/Services/OpenAccessLicense), applicable to the online version of the article only. Usage and distribution for commercial purposes requires written permission. years. In 626 patients (59\%), 3 or more domains were impaired. All domains were independently associated with mortality, with the highest hazard for the somatic domain (HR 3.7 [1.7-8.0]) and the lowest hazard for the mental domain (HR 1.5 [1.1-12.0]). In addition, accumulation of impaired domains showed a gradually increased mortality risk, ranging from HR $2.2(0.8-6.1)$ for 2 domains to HR 9.6 (3.724.7) for all 5 domains impaired. Conclusions: This study provides evidence that impairment in multiple geriatric domains is highly prevalent and independently and cumulatively associated with mortality in an outpatient geriatric setting.

(C) 2021 The Author(s)

Published by S. Karger AG, Basel

\section{Introduction}

Over the next decades, the proportion of the world's population older than 60 years doubles, whereas the population older than 80 years triples [1]. As a result, the proportion of older patients needing health care will rapidly increase. Older patients are at increased risk of several adverse health outcomes and prognosis is closely associated with the presence of concomitant disease and the 
degree of mental, nutritional, physical, and social impairment [2-4].

It is, therefore, important to assess older patients, particularly frail older patients, with a comprehensive geriatric assessment (CGA) [5]. The CGA is an important instrument within geriatric medicine, systematically assessing different geriatric domains [6, 7]. Guidelines recommend to include questionnaires and measurements of at least somatic, mental, cognitive, physical, and social functions to produce an inventory of health problems, which will serve to define individualized care goals and advance care planning $[8,9]$.

Previous research has shown the value of separate geriatric domains in predicting adverse health outcomes in hospitalized patients or in disease-specific populations, such as oncology or heart failure populations [10-15]. To the best of our knowledge and somewhat surprising, however, the relation between different geriatric domain deficits and mortality in older patients referred to an outpatient geriatric clinic has not been studied. We, therefore, investigated the distribution and accumulation of different geriatric domain deficits and their association with overall mortality in an ongoing longitudinal cohort of an outpatient geriatric clinic.

\section{Materials and Methods}

\section{Patients}

The Amsterdam Ageing Cohort is a novel ongoing longitudinal cohort study of the outpatient geriatric clinic at the Amsterdam University Medical Center, location VU University Medical Center. In this article, we present the first results of the cohort. All patients were referred to the outpatient day clinic for medical care with different reasons for referral, such as memory problems, falls, and decline in physical function. From this cohort, we included all patients who had a baseline visit between February 2016 and December 2019 and provided informed consent, resulting in 1,055 patients. We used no other exclusion criteria.

At baseline, all patients received a standardized and multidisciplinary GCA, administered by trained nurses and medical staff. All screening tools are widely used and, in our cohort, performed as part of routine medical care. In those who were referred with memory problems, additional neuropsychological examination and brain MRI was performed. Diagnoses were made in a multidisciplinary consensus meeting. The protocol of our cohort is approved by the local Medical Ethical Committee, all patients provided written informed consent.

\section{Assessment of Geriatric Domains}

We defined 5 geriatric domains: somatic, mental, nutritional, physical, and social domain. We scored the separate domains impaired as yes/no according to predefined cutoff values using individual components as described below and calculated the total sum of domains impaired (0-5).

Mortality Risk and Its Association with Geriatric Domain Deficits
Somatic Domain

We calculated the Charlson Comorbidity Index (CCI) based on medical history and medication used [16]. A score at or above 5 points was considered abnormal $[17,18]$. As part of the CCI, we used renal function to score for the presence of moderate to CKD. To calculate renal function, we used the Modified Diet in Renal Disease (MDRD) study equation until first of April 2017, and afterward we used the Chronic Kidney Disease Epidemiology Collaboration method $[19,20]$. Polypharmacy was defined as the (chronic) use of 5 or more drugs [21]. We scored the somatic domain impaired if the CCI was scored above cutoff and/or polypharmacy was present.

\section{Mental Domain}

Cognitive functions were assessed at baseline with the Mini Mental State Examination (MMSE) for global cognitive functioning; a score below 24 was considered abnormal [22]. The Geriatric Depression Scale (GDS-15) was used to detect depressive symptoms; a score at or above 6 points was considered abnormal, as it indicates a possible depression [23]. We scored the mental health domain impaired if the MMSE and/or GDS was abnormal.

\section{Nutritional Domain}

The Short-Form Mini Nutritional Assessment (MNA) was used to identify patients who were at risk for malnutrition; a score below 12 indicates risk of malnutrition [24]. BMI was calculated by measuring the weight and height of the patient; a score below 18.5 was considered abnormal [25]. We scored the nutritional domain impaired if the MNA and/or BMI was below cutoff.

\section{Physical Domain}

Maximal handgrip strength (HGS; 4 measures average; 2 measures per hand) was measured using a Jamar hand-held dynamometer [26]. Grip strength below $30 \mathrm{~kg}$ for men and below $20 \mathrm{~kg}$ for women is considered abnormal [27]. Gait speed was measured in $\mathrm{m} / \mathrm{s}$, calculated from time needed to complete a distance of $4 \mathrm{~m}$ at usual pace. Use of a walking cane or a walker was allowed when needed. When a patient was unable to walk, gait speed was recorded as 0 . A gait speed below 0.8 is considered abnormal [28]. The ability to perform activities of daily living (ADL) independently was assessed using the Katz ADL questionnaire, where a score at or above 1 is abnormal[29]. We scored the functional domain impaired if at least 1 of the 3 measures (handgrip strength, gait speed and/or ADL) was abnormal.

\section{Social Domain}

We used information on living situation and needed care. We defined the social domain impaired if the patient was living in a nursing home or if professional care at home (for ADL support) was needed.

\section{Mortality}

For each patient, we obtained information on all-cause mortality (died yes/no with the date of death) from the Dutch municipal population register. This register was searched on January 22, 2020. Causes of death cannot be determined from this municipal registry. From these data, we derived our outcome measure: overall mortality. For the overall mortality, we included all patients and defined follow-up duration as time between date of baseline visit and date of death, or if alive, between date of baseline visit and January 22, 2020. 
Table 1. Baseline characteristics for total group and stratified per amount of domains impaired



Follow-up in years: from baseline visit to either date of death, or in case of alive, date of check Dutch municipal population register. Data are presented as mean \pm SD, unless otherwise specified. The presence of an impaired domain is defined in Table 2. HGS, handgrip strength; GP, general practitioner; CCI, Charlson Comorbidity Index; MMSE, Mini Mental State Examination; GDS, Geriatric Depression Scale; MNA, Short-Form Mini Nutritional Assessment; ADL, activities of daily living.

\section{Statistical Analyses}

Statistical analyses were performed using SPSS version 22 (IBM, Armonk, NY, USA). A $p$ value $<0.05$ was considered statistically significant. Baseline characteristics were calculated for the total population and according to the sum of impaired geriatric domains. Next, the prevalence of impaired domains and its individual components were stratified for alive or dead. Continuous and dichotomized variables were compared using parametric and nonparametric tests where appropriate. To visualize the overlap and accumulation of domains impaired, a Venn diagram was created [30] and we assessed correlations using Cramer's V.

Next, we used pattern analyses to explore the amount and randomness of missing data. Since missing data were at random but not completely at random, we imputed the missing individual components of the geriatric domains using multiple imputations with 15 imputation cycles. With the imputed individual components, we recreated the domains. Then, Cox proportional hazard models were used to assess associations between determinants and mortality risk, taking into account time to death using the pooled results of the 15 imputations. First, we analyzed the individual components used to create the geriatric domains (dichotomized according to Table 2). Second, we examined the association between the separate domains (somatic, mental, nutritional, physical, and social) and mortality risk. Third, we assessed the influence of the accumulation (i.e., sum) of domains impaired on mortality risk, using the presence of 0 or 1 domain impaired as the reference category. We performed all analyses adjusted for age and sex, and in addition for age $^{2}$. In addition, we repeated above Cox propor- 
Table 2. Definition of domains and amount of impaired components/domains, stratified for mortality

\begin{tabular}{|c|c|c|c|c|}
\hline \multirow[t]{2}{*}{ Domain } & \multirow[t]{2}{*}{ Impaired if } & \multicolumn{2}{|c|}{ Impaired in $n(\%)$} & \multirow[t]{2}{*}{$p$ value } \\
\hline & & alive $n=883$ & dead $n=172$ & \\
\hline Somatic & & $720(82)$ & $165(96)$ & $<0.001$ \\
\hline CCI [10] & $>4$ points & $535(61)$ & $142(83)$ & $<0.001$ \\
\hline Polypharmacy [15] & $>5$ number of prescriptions & $575(65)$ & $133(77)$ & 0.002 \\
\hline Mental & & $487(56)$ & $108(65)$ & 0.032 \\
\hline MMSE [16] & $<24$ points & $345(40)$ & $85(52)$ & 0.006 \\
\hline GDS [17] & $>5$ points & $221(26)$ & $45(30)$ & 0.336 \\
\hline Nutritional & & $415(48)$ & $92(58)$ & 0.018 \\
\hline MNA [18] & $<12$ points & $415(50)$ & $90(63)$ & 0.004 \\
\hline BMI [19] & $<18.5 \mathrm{~kg} / \mathrm{m}^{2}$ & $16(2)$ & $10(7)$ & 0.001 \\
\hline Physical & & $580(66)$ & $140(86)$ & $<0.001$ \\
\hline HGS [21] & $<30 \mathrm{~kg} \mathrm{M},<20 \mathrm{~kg} \mathrm{~F}$ & $352(44)$ & $97(70)$ & $<0.001$ \\
\hline Gait speed [22] & $<0.8 \mathrm{~m} / \mathrm{s}$ & $318(39)$ & $86(63)$ & $<0.001$ \\
\hline Katz ADL [23] & $\geq 1$ points & $279(34)$ & $84(56)$ & $<0.001$ \\
\hline Social & & $196(22)$ & $83(48)$ & $<0.001$ \\
\hline Living situation & In nursing home & $30(3)$ & $18(11)$ & $<0.001$ \\
\hline Needed care & Professional care present & $186(22)$ & $81(48)$ & $<0.001$ \\
\hline
\end{tabular}

Group differences between alive or dead were calculated using $\chi^{2}$ test. A $p$ value $<0.05$ was considered statistically significant. HGS, handgrip strength; CCI, Charlson Comorbidity Index; MMSE, Mini Mental State Examination; GDS, Geriatric Depression Scale; MNA, Short-Form Mini Nutritional Assessment; ADL, activities of daily living.

tional hazard models using nonimputed data yielding comparable results (see online suppl. Table; for all online suppl. material, see www.karger.com/doi/10.1159/000512048). Finally, for illustrative purposes, we created Kaplan-Meier curves for the accumulation of domains impaired and report log rank tests, using the raw data without imputation. Data are represented as hazard ratios (HR) with accompanying 95\% confidence intervals (CIs).

\section{Results}

Table 1 shows the baseline characteristics. After a mean follow-up duration of $1.7 \pm 1.1$ years, $172(16 \%)$ patients had died. In 687 (65\%) of the total group, the primary reason for referral was cognitive problems, whereas 365 (35\%) were referred for somatic problems. The majority of patients had 3 or more domains impaired $(n=$ 626 [59\%]); this was similar for patients referred for cognitive problems as for somatic problems (58 and 61\%). With increasing amount of impaired domains, patients were older and more often female. Further, with increasing amount of impaired domains, all individual components of the somatic, mental, nutritional, physical, and social domain scores showed a tendency to deteriorate (Table 1).

To assess the impaired domains, their overlap, and accumulation, we created a Venn diagram. Figure 1 shows an even overlap and accumulation of the separate domains. The correlations between the impaired domains were low (Cramer's V ranging from 0.10 to 0.30 ).

Table 2 shows the amount of impaired components and domains, stratified for alive or dead. All domains, and the components used to construct them, were more often impaired in patients who had died. Only the proportion of patients with an abnormal GDS did not differ between patients alive and dead.

Next, we assessed the association between the CGA and mortality using Cox proportional hazard models (Table 3). First, we studied the association between the (dichotomized) individual components and mortality. We found all components, except a GDS $>5$, to be associated with mortality. Second, when we assessed the association between the separate domains and mortality, we found all domains to be associated with an increased mortality risk. The somatic domain showed the highest 
Table 3. Cox proportional hazard models; influence of individual dichotomized components and separate domains on mortality

\begin{tabular}{|c|c|c|}
\hline & \multicolumn{2}{|c|}{ Adjusted for age and sex } \\
\hline & $\begin{array}{l}\text { Individual } \\
\text { component }\end{array}$ & $\begin{array}{l}\text { Separate } \\
\text { domain }\end{array}$ \\
\hline \multicolumn{3}{|l|}{ Somatic } \\
\hline Domain impaired & & $3.7(1.7-8.0)^{*}$ \\
\hline$>5$ number of prescriptions & $1.7(1.2-2.4)^{*}$ & \\
\hline $\mathrm{CCI}>4$ & $2.2(1.4-3.3)^{*}$ & \\
\hline \multicolumn{3}{|l|}{ Mental } \\
\hline Domain impaired & & $1.5(1.1-2.0)^{*}$ \\
\hline MMSE $<24$ & $1.5(1.1-2.0)^{*}$ & \\
\hline GDS $>5$ & $1.3(0.9-1.8)$ & \\
\hline \multicolumn{3}{|l|}{ Nutritional } \\
\hline Domain impaired & & $1.9(1.3-2.6)^{*}$ \\
\hline MNA $<12$ & $1.9(1.3-2.6)^{*}$ & \\
\hline BMI $<18.5$ & $3.1(1.6-6.2)^{*}$ & \\
\hline \multicolumn{3}{|l|}{ Physical } \\
\hline Domain impaired & & $2.3(1.4-3.9)^{*}$ \\
\hline HGS, female $<20 \mathrm{~kg}$ & $1.8(1.1-3.0)^{*}$ & \\
\hline HGS, male $<30 \mathrm{~kg}$ & $1.7(1.0-3.0)^{*}$ & \\
\hline Gait speed $<0.8 \mathrm{~m} / \mathrm{s}$ & $1.9(1.4-2.8)^{*}$ & \\
\hline Katz ADL $\geq 1$ & $1.9(1.4-2.6)^{*}$ & \\
\hline \multicolumn{3}{|l|}{ Social } \\
\hline Domain impaired & & $2.5(1.8-3.4)^{*}$ \\
\hline Living in nursing home & $3.1(1.9-5.1)^{*}$ & \\
\hline Professional care at home & $2.5(1.8-3.5)^{*}$ & \\
\hline
\end{tabular}

Data are presented as $\mathrm{HR}(95 \% \mathrm{CI})$ for the presence of an impaired domain (as defined in Table 2) and the dichotomous variable, for mortality, using pooled data of 15 imputations. HGS, handgrip strength; HR, hazard ratio; CI, confidence interval; CCI, Charlson Comorbidity Index; MMSE, Mini Mental State Examination; GDS, Geriatric Depression Scale; MNA, Short-Form Mini Nutritional Assessment; ADL, activities of daily living. ${ }^{*} p<$ 0.05 .

hazard (HR 3.7 [1.7-8.0]) and the mental domain the lowest hazard (HR $1.5[1.1-2.0]$ ).

Finally, we examined the effect of the accumulation of impaired domains on mortality. More domains impaired resulted in a higher hazard of mortality ( $p$ for trend $<0.001)$. Figure 2 shows the corresponding Kaplan-Meier survival curve and HRs. Since age is an important and strong confounder, we repeated the analyses with age ${ }^{2}$ in the models, after which the effect sizes in Table 3 and Figure 2 remained similar (results not shown).

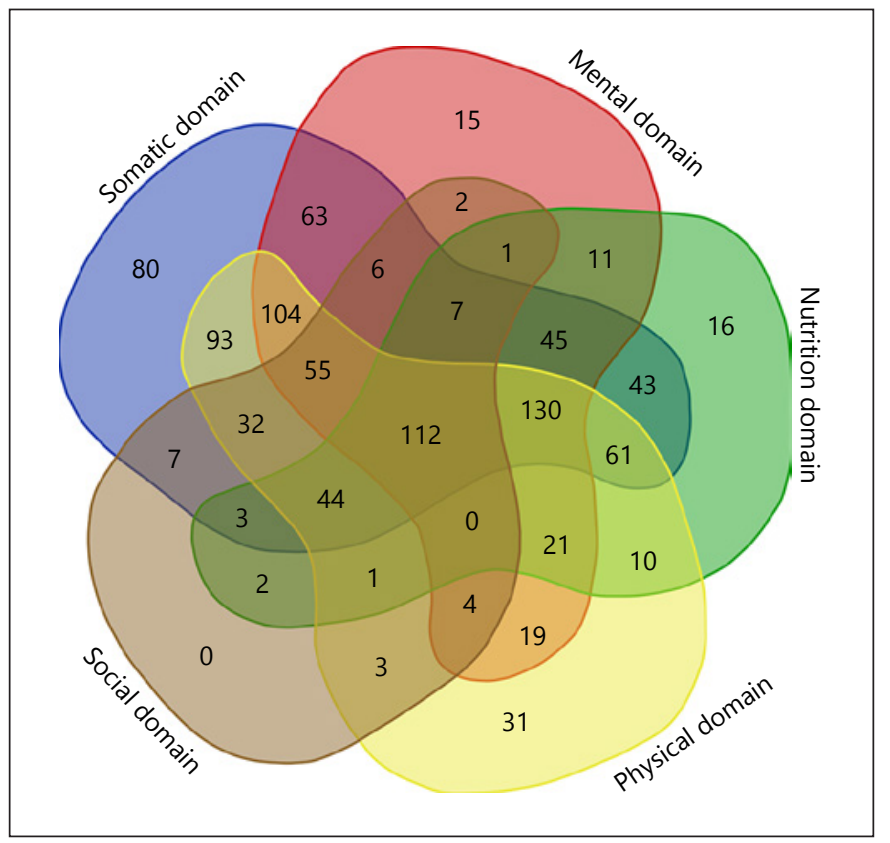

Fig. 1. Venn diagram showing the domains impaired, their overlap, and accumulation. The diagram was created using [30]. The presence of an impaired domain is defined in Table 2.

\section{Discussion/Conclusion}

In this longitudinal cohort of an outpatient geriatric clinic, we not only show that impairment in multiple geriatric domains is highly prevalent but also demonstrate that geriatric domain deficits are independently and cumulatively associated with mortality. These findings illustrate the added value of the CGA in older patients referred to a geriatric outpatient clinic. Therefore, the CGA does not only function as an inventory of different health problems and related treatment options but could also be useful to determine the prognosis of older patients.

Our study adds to earlier studies by focusing on a broad range of geriatric domains and their accumulation in an outpatient geriatric population. To date, available literature focused mainly on the association of separate geriatric domains with mortality in specific patient categories, for example, oncology, renal or heart failure patients $[12,13,15]$, hospitalized geriatric patients $[10,11$, $14]$, or solely on individual components [2, 31-33], with inconsistent findings. As a result, knowledge on the relation between a CGA, its geriatric domains and mortality in an outpatient geriatric population has remained sparse. We show that a multi-domain approach is not only appropriate in disease-specific populations, with known 
Fig. 2. Kaplan-Meier survival curve per total domains impaired, for total group (log rank <0.001). Observed survival is analyzed by Kaplan-Meier using nonimputed data, whereas the association of amount of domains and mortality is analyzed using Cox models, adjusted for age and sex, taking 0 and 1 domains impaired as reference, using pooled data of 15 imputations. ${ }^{*} p<$ 0.05 .

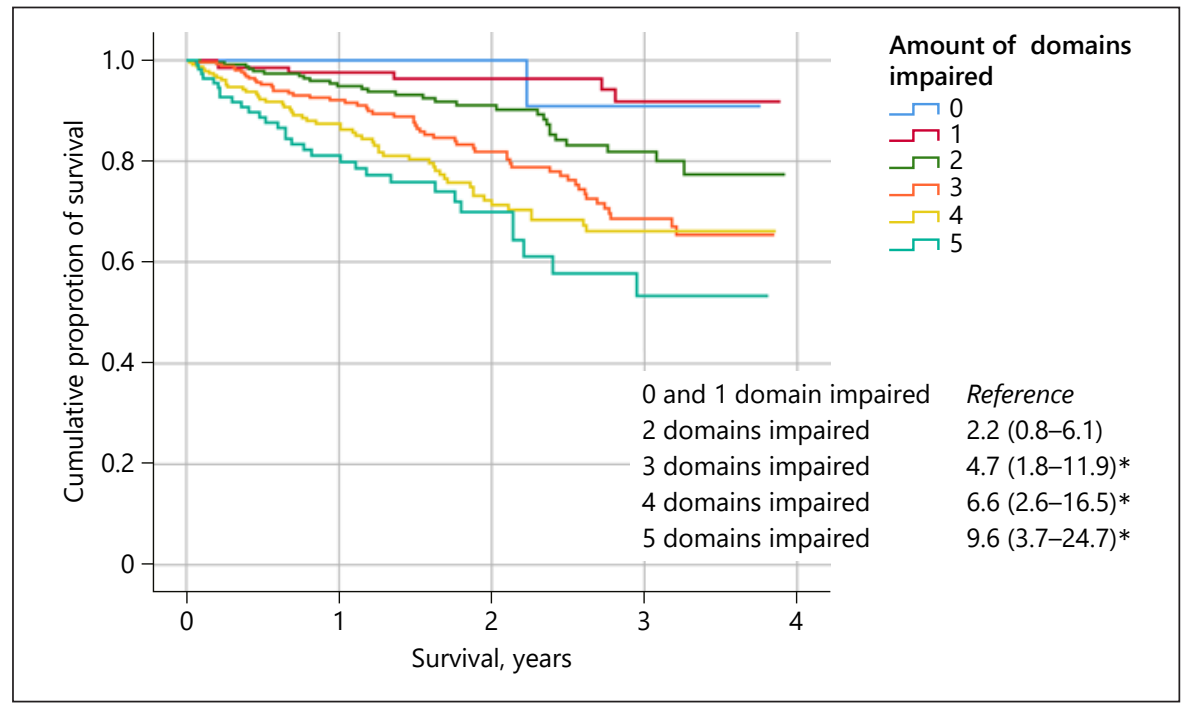

vulnerability to negative outcomes but also in a very heterogeneous population, referred for various reasons, of outpatient older subjects.

These results have important clinical implications. A multi-domain assessment helps to detect (modifiable) vulnerabilities, but also helps to detect a patient's vulnerability, irrespective of age and reason for referral. It informs physicians and patients about therapeutic priorities [34]. While patients with single domain deficits are in need of a problem-based disease-orientated approach, accumulation of domain deficits helps physicians to shift toward a more "goal-oriented, integrated approach" [35]. Identifying the most vulnerable patients (e.g., those with 3 or more impaired domains impaired) with a short life expectancy can subsequently lead to focus on, for example, advance care planning and an optimization of provided care, resulting in improvement of quality of life [3].

One can wonder if age did not drive the observed associations since age is an important and strong predictor of death. However, effect sizes remained comparable after correcting for age and even after correcting for age ${ }^{2}$. Similarly, a specific domain, such as the physical domain containing measures of frailty, could have primarily caused the results [36], but again our results remained more or less similar when correcting for physical impairment. Finally, contrary to our expectations, presence of a GDS $>5$ was not associated with mortality. Yet, previous studies have shown an age-dependent association of GDS and mortality, with the strongest association at younger age $[37,38]$. With increasing age, impairment in other do-

Mortality Risk and Its Association with Geriatric Domain Deficits mains related to mortality, seem to dilute this association [39]. These observations together with poor overlap of geriatric domain deficits as illustrated by our Venn diagram emphasize the clinical relevance of a multi-domain assessment approach in geriatric outpatients.

This study has several strengths. In contrast to previous literature, we were able to include a substantial, reallife, geriatric outpatient population and focused on a wide scope of domains and mortality. All patients underwent a standardized workup, including commonly used instruments, derived as part of medical routine care. This resulted in a well-defined phenotyping of the included patients, referred for cognitive as well as somatic problems. Furthermore, although unintentionally, we obtained our information on mortality from the municipal population register before COVID-19 caused excess mortality.

This study also has limitations. First of all, no guideline is available on how to define geriatric domains $[5,6]$. Our construction of domains can thus be considered as somewhat arbitrary. This is especially true for the social domain. Since questionnaires (e.g., EDIZ, ZARIT, or CSI) providing more detailed information on the social domain, are missing, our approach should be considered as proxy for the social domain [40]. However, for the definition of the other geriatric domains, we used tests that are widely available and easily applicable, with acknowledged cutoff points, and commonly used in clinical practice [6]. Second, we lacked information on adverse health outcome measurers other than death, such as functional decline, hospital admission, or institutionalization. Third, 
our follow-up duration was on average 1.7 years. A different pattern of associated domains and mortality might have been found if our follow-up duration had been longer.

In conclusion, this study provides longitudinal evidence that impairment in multiple geriatric domains is highly prevalent and, irrespective of age, cumulatively associated with mortality in an outpatient geriatric setting. Depending on the number of impaired domains, this approach helps to make considerations on treatment decisions, care programs, or advanced care planning all aimed at improvement of quality of life and reducing unnecessary care consumption.

\section{Acknowledgements}

The Amsterdam Ageing Cohort could not exist for the help of many students and supporting staff. The authors would in particular like to thank Greetje Asma, Gerda Bolink, Anouk Burger, Elske Gieteling, Gooke Lagaay, Arenda Mank, Petra Moens, Astrid Verburg-Bakker, Barbara Verhaar, and Kathelijn Versteeg for their effort.

\section{Statement of Ethics}

The protocol of our cohort is approved by the Medical Ethical Committee of the Amsterdam UMC, location VUmc under protocol number 2017.148, and all patients provided written informed consent.

\section{Conflict of Interest Statement}

Hanneke F.M. Rhodius-Meester performs contract research for Combinostics, all funding is paid to her institution. Sara A.J. van de Schraaf, Mike J.L. Peters, Emma E.F. Kleipool, Marijke C. Trappenburg, and Majon Muller report no disclosures.

\section{Funding Sources}

The authors did not receive any funding.

\section{Author Contributions}

Hanneke F.M. Rhodius-Meester drafted the manuscript and performed the analyses, Sara A.J. van de Schraaf acquired the data and performed the analyses, Mike J.L. Peters, Emma E.F. Kleipool, Marijke C. Trappenburg, and Majon Muller contributed to the concept and design of the study. All authors contributed to critical revision of the manuscript.

\section{References}

1 WHO. Factsheet ageing and health. 2018.

2 Tamura Y, Ishikawa J, Fujiwara Y, Tanaka M, Kanazawa N, Chiba Y, et al. Prevalence of frailty, cognitive impairment, and sarcopenia in outpatients with cardiometabolic disease in a frailty clinic. BMC Geriatr. 2018 Nov 6; 18(1):264.

3 Mazya AL, Garvin P, Ekdahl AW. Outpatient comprehensive geriatric assessment: effects on frailty and mortality in old people with multimorbidity and high health care utilization. Aging Clin Exp Res. 2019 Apr;31(4): $519-25$.

4 Rhodius-Meester HFM, Tijms BM, Lemstra AW, Prins ND, Pijnenburg YAL, Bouwman F, et al. Survival in memory clinic cohort is short, even in young-onset dementia. J Neurol Neurosurg Psychiatry. 2019 Jun;90(6):726-8.

5 Epstein AM, Hall JA, Besdine R, Cumella E Jr, Feldstein M, McNeil BJ, et al. The emergence of geriatric assessment units. The "new technology of geriatrics". Ann Intern Med. 1987 Feb;106(2):299-303.

6 Parker SG, McCue P, Phelps K, McCleod A, Arora S, Nockels K, et al. What is comprehensive geriatric assessment (CGA)? An umbrella review. Age Ageing. 2018 Jan 1;47(1):14955.
7 Aliberti MJR, Cenzer IS, Smith AK, Lee SJ, Yaffe K, Covinsky KE. Assessing risk for adverse outcomes in older adults: the need to include both physical frailty and cognition. J Am Geriatr Soc. 2019 Mar;67(3):477-83.

8 Molag ML, Golüke-Willemse GAM, Arends AJ, van Barneveld TA. De richtlijn 'Comprehensive geriatric assessment'. Nederlands tijdschrift voor geneeskunde. 2011;155:A3815.

9 British Geriatric Society. Comprehensive Geriatric Assessment Toolkit for primay care practicioners. 2019.

10 Martínez-Velilla N, Ibáñez-Beroiz B, Cambra-Contin K, Alonso-Renedo J. Is comprehensive geriatric assessment a better 1-year mortality predictor than comorbidity and prognostic indices in hospitalized older adults? J Am Geriatr Soc. 2013 Oct;61(10): 1821-3.

11 Avelino-Silva TJ, Farfel JM, Curiati JA, Amaral JR, Campora F, Jacob-Filho W. Comprehensive geriatric assessment predicts mortality and adverse outcomes in hospitalized older adults. BMC Geriatr. 2014 Dec 3;14(1):129.

12 Huisman MG, Kok M, de Bock GH, van Leeuwen BL. Delivering tailored surgery to older cancer patients: preoperative geriatric assessment domains and screening tools: a systematic review of systematic reviews. Eur J Surg Oncol. 2017 Jan;43(1):1-14.
13 Bruijnen CP, van Harten-Krouwel DG, Koldenhof JJ, Emmelot-Vonk MH, Witteveen $\mathrm{PO}$. Predictive value of each geriatric assessment domain for older patients with cancer: a systematic review. J Geriatr Oncol. 2019 Nov; 10(6):859-73.

14 Gregersen M, Hansen TK, Jørgensen BB, Damsgaard EM. Frailty is associated with hospital readmission in geriatric patients: a prognostic study. Eur Geriatr Med. 2020 Oct; 11(5):783-92.

15 Kleipool EEF, Wiersinga JHI, Trappenburg MC, van Rossum AC, van Dam CS, Liem SS, et al. The relevance of a multidomain geriatric assessment in older patients with heart failure. ESC Heart Fail. 2020 Jun;7(3):1264-72.

16 Charlson M, Szatrowski TP, Peterson J, Gold $\mathrm{J}$. Validation of a combined comorbidity index. J Clin Epidemiol. 1994 Nov;47(11):124551.

17 Frenkel WJ, Jongerius EJ, Mandjes-van Uitert MJ, van Munster BC, de Rooij SE. Validation of the Charlson Comorbidity Index in acutely hospitalized elderly adults: a prospective cohort study. J Am Geriatr Soc. 2014 Feb;62(2): 342-6.

18 Soh CH, Ul Hassan SW, Sacre J, Maier AB. Morbidity measures predicting mortality in inpatients: a systematic review. J Am Med Dir Assoc. 2020 Apr;21(4):462-e7. 
19 Levey AS, Bosch JP, Lewis JB, Greene T, Rogers N, Roth D, et al. A more accurate method to estimate glomerular filtration rate from serum creatinine: a new prediction equation. Anna Intern Med. 1999;130(6):461-70.

20 Levey AS, Stevens LA, Schmid CH, Zhang YL, Castro AF 3rd, Feldman HI, et al. A new equation to estimate glomerular filtration rate. Ann Intern Med. 2009 May 5;150(9):604-12.

21 Masnoon N, Shakib S, Kalisch-Ellett L, Caughey GE. What is polypharmacy? A systematic review of definitions. BMC Geriatr. 2017 Oct 10;17(1):230.

22 Folstein MF, Folstein SE, McHugh PR. "Minimental state". A practical method for grading the cognitive state of patients for the clinician. J Psychiatr Res. 1975 Nov;12(3):189-98.

23 Sheikh JI, Yesavage JA. Geriatric Depression Scale (GDS): recent evidence and development of a shorter version. Clin Gerontol. 1986;5(1-2):165-73.

24 Rubenstein LZ, Harker JO, Salvà A, Guigoz Y, Vellas B. Screening for undernutrition in geriatric practice: developing the short-form mini-nutritional assessment (MNA-SF). J Gerontol A Biol Sci Med Sci. 2001 Jun;56(6): M366-72.

25 Cederholm T, Bosaeus I, Barazzoni R, Bauer J, Van Gossum A, Klek S, et al. Diagnostic criteria for malnutrition: an ESPEN consensus statement. Clin Nutr. 2015 Jun;34(3):335-40.

26 Reijnierse EM, de Jong N, Trappenburg MC, Blauw GJ, Butler-Browne G, Gapeyeva H, et al. Assessment of maximal handgrip strength: how many attempts are needed? J Cachexia Sarcopenia Muscle. 2017 Jun;8(3):466-74.
27 Lauretani F, Russo CR, Bandinelli S, Bartali B, Cavazzini C, Di Iorio A, et al. Age-associated changes in skeletal muscles and their effect on mobility: an operational diagnosis of sarcopenia. J Appl Physiol. 2003 Nov;95(5):1851-60.

28 Odden MC, Peralta CA, Haan MN, Covinsky KE. Rethinking the association of high blood pressure with mortality in elderly adults: the impact of frailty. Arch Intern Med. 2012 Aug 13;172(15):1162-8.

29 Katz S. Assessing self-maintenance: activities of daily living, mobility, and instrumental activities of daily living. J Am Geriatr Soc. 1983 Dec;31(12):721-7.

30 Calculate and draw custom Venn diagrams. VIB/UGent Bioinformatics \& Evolutionary Genomics; Gent, Belgium. Date of consultation and creation: 2020 May 16. Available from: http://bioinformatics.psb.ugent.be/ webtools/Venn/.

31 Kara O, Canbaz B, Kizilarslanoglu MC, Arik G, Sumer F, Aycicek GS, et al. Which parameters affect long-term mortality in older adults: is comprehensive geriatric assessment a predictor of mortality? Aging Clin Exp Res. 2017 Jun;29(3):509-15.

32 Ritt M, Ritt JI, Sieber CC, Gassmann KG. Comparing the predictive accuracy of frailty, comorbidity, and disability for mortality: a 1 -year follow-up in patients hospitalized in geriatric wards. Clin Interv Aging. 2017;12: 293-304.

33 Freud T, Punchik B, Kagan E, Barzak A, Press Y. Orthostatic hypotension and overall mortality in 1050 older patients of the outpatient comprehensive geriatric assessment unit Geriatr Gerontol Int. 2018 Jul;18(7):1009-17.
34 Blom J, den Elzen W, van Houwelingen AH, Heijmans M, Stijnen T, Van den Hout W, et al. Effectiveness and cost-effectiveness of a proactive, goal-oriented, integrated care model in general practice for older people. A cluster randomised controlled trial: integrated systematic care for older people: the ISCOPE study. Age Ageing. 2016 Jan;45(1):3041.

35 Kleipool EEF, Peters MJL, Muller M. Letter by Kleipool et al regarding article, "primary prevention with statin therapy in the elderly: new meta-analyses from the contemporary JUPITER and HOPE-3 randomized trials". Circulation. 2017 Oct 10;136(15):1456-7.

36 Hubbard RE, Maier AB, Hilmer SN, Naganathan V, Etherton-Beer C, Rockwood K. Frailty in the face of COVID-19. Age Ageing. 2020 Jul 1;49(4):499-500.

37 Cuijpers P, Vogelzangs N, Twisk J, Kleiboer A, Li J, Penninx BW. Differential mortality rates in major and subthreshold depression: meta-analysis of studies that measured both. Br J Psychiatry. 2013;202(1):22-7.

38 Ludvigsson M, Marcusson J, Wressle E, Milberg A. Morbidity and mortality in very old individuals with subsyndromal depression: an 8-year prospective study. Int Psychogeriatr. 2019;31(11):1569-79.

39 Vaughan L, Corbin AL, Goveas JS. Depression and frailty in later life: a systematic review. Clin Interv Aging. 2015;10:1947-58.

40 Oud FMM, de Rooij SEJA, Arends AJ, Emmelot-Vonk MH, Melis RJF, Mooijaart SP, et al. [Assessment instruments in frail older patients; a call for more standardisation]. Ned Tijdschr Geneeskd. 2019 Nov 12;163:163. 